\title{
A Study of the Wellbeing of Siblings of Children with Autism Spectrum Disorders: Sibling Efficacy, Positive and Negative Affect, and Coping Strategies
}

\author{
Emily Anne Habelrih ${ }^{1}$, Richard Edward Hicks ${ }^{1} \&$ Daisy Mary Vanstone ${ }^{1}$ \\ ${ }^{1}$ School of Psychology, Bond University, Robina, Queensland, Australia \\ Correspondence: Richard E. Hicks, School of Psychology, Faculty of Society and Design, Bond University, \\ Robina, Queensland-4229, Australia
}

Received: April 30, 2018

Accepted: May 18, 2018

Online Published: May 28, 2018

doi:10.5539/ijps.v10n2p102

URL: https://doi.org/10.5539/ijps.v10n2p102

\begin{abstract}
Autism spectrum disorders (ASD) include pervasive developmental disorders characterised by communication deficits, difficulty with social understanding, and repetitive behaviors. Few studies have compared the efficacy, affect, and coping strategies of siblings of typically developing children with siblings of children with ASD. Typically developing siblings are understood to be at an increased risk of externalising and internalising problems. The current study examined whether siblings of children with ASD differed in levels of efficacy, affect, and coping from siblings of typically developing children. Participants (156) included an Australia-wide sample involving 82 siblings of children with ASD, and 74 siblings of typically developing individuals. Participants completed The Self-Efficacy Scale for Children (assessing social, emotional, and academic efficacy), the Positive and Negative Affect Scales, the Brief COPE Scale, and other scales as part of the larger study. Results showed that ASD siblings reported lower scores on emotional efficacy, social efficacy, and positive affect, and higher negative affect, than did the comparison group siblings. However, no significant differences were found in coping strategies or academic efficacy between the ASD siblings and the typically developing siblings. Consistent with earlier research findings, there are perceived negative effects or risks from being a sibling of an individual with ASD, suggesting support interventions may assist the development of emotional and social efficacy and increased positive affect for these individuals.
\end{abstract}

Keywords: autism spectrum disorder, siblings, affect, efficacy, coping

\section{Introduction}

\subsection{Background}

Autism spectrum disorders (ASD) are pervasive developmental disorders that are characterized by social and communication deficits (American Psychiatric Association, 2013). ASD is further characterized by repetitive, ritualistic patterns of behavior, interests, food choices, and activities. In addition, cognitive deficits, challenging behaviors such as self-harm, aggression, a strong need for routine, and sensory sensitivity are other common characteristics of ASD (Hesse, Danko, \& Budd 2013). The prevalence of ASD appears to be rising, with the Australian Bureau of Statistics (ABS) estimating that 164,000 Australians had autism in 2015, representing 1 in 150 Australians.

As the prevalence of ASD continues to rise, so does the need to evaluate the impact of associated difficulties on both the diagnosed child and the immediate family. From a family systems' perspective, the psychological well-being of one family member is likely to affect the well-being of other individuals within this system, such as mother-father relationships, parent-child relationships, and sibling relationships (Cridland, Jones, Magee, \& Caputi, 2013; Giallo, Roberts, Emerson, Wood, \& Gavidia-Payne, 2014; Griffith, Hastings, \& Petalas, 2014; Kovshoff, Cebula, Tsai, \& Hastings, 2017). Furthermore, although having a family member with an illness or disability presents challenges, the unique and complex combination of impairments associated with ASD places parents and siblings at a higher risk for psychological difficulties and distress (Glasberg, 2000). This risk is due to the extent of disruption to the family system, having fewer recreational activities as a family, and distress regarding the future of a child with ASD (Macks \& Reeve, 2007). The unique communication and behavioral difficulties experienced by children with ASD make family outings challenging (Kaminsky \& Dewey, 2002), 
likely resulting in further ruptures in the family system. Accordingly, the wellbeing overall of family members is at risk, and especially perhaps also among siblings. However, only a few studies exist (see below) that have examined specific effects on members of the family, especially siblings, in relation to efficacy, affect and coping strategies used.

The current study therefore examined differences in wellbeing in terms of efficacy, affect, and overall coping strategies between siblings of non-diagnosed children and siblings of ASD diagnosed children, in an Australian-based sample. For the purposes of the current study, the term "well-being" was interpreted as a general and multifaceted descriptor comprising of behavioral, emotional, and cognitive components. The current study measured specific variables (efficacy, affect, and coping) that were thought to be indicative of overall psychological well-being. To exhibit greater positive reports on these components is indicative of greater levels of well-being (Linley, Maltby, Wood, Osborne, \& Hurling, 2009). That is, the current study aimed to address the factors that contribute to sibling well-being by determining whether siblings of individuals with ASD differed in levels of efficacy, affect, and coping from siblings of typically developing individuals. First, we discuss Family Systems Theory as underpinning much observation and research on family relationships.

\subsection{Family Systems Theory}

Family Systems Theory (Cridland, Jones, Magee, \& Caputi, 2013) includes several basic assumptions: (a) family characteristics are inputs into the family system, to which the family responds, and from which outputs (i.e., affectionate behaviors) are produced, (b) the family is a whole system and is affected by the relationships between family members, and (c) boundaries exist between family subsystems (i.e., parental, marital, and sibling) and with the outside world. That is, in clarification of the final assumption, the relationship between one subsystem, such as that between siblings, can impact the entire family system. Overall, it is this family system that provides children with their first interactions with others. For families comprising of children with ASD, the sibling subsystem is of heightened importance, as the sibling relationship is the first and most intense peer relationship (Cridland et al., 2013). Typical or 'normal' sibling relationships change over time and provide opportunities for siblings to experience sharing, companionship, and rivalry. Some sibling pairs experience warm, supportive relationships, whereas others experience conflicts and isolation (Cridland et al., 2013). Due to the complex nature of ASD, siblings may receive reduced parental attention and support, which may lead to resentment towards their sibling with ASD (Nordone, 2014). Overall, siblings of individuals with ASD are clearly affected by family systems but these in turn are affected by broader systems of culture indicating multiple factors impacting on siblings living in families (see Kovshoff, Cebula, Tsai, \& Hastings, 2017).

Many researchers have explored sibling relationships, adjustment, and outcomes when a sibling has an ASD diagnosis. Meadan, Stoner, and Angell (2010) reviewed literature pertaining to the emotional, social, and behavioral adjustment of siblings of children with ASD. This analysis revealed mixed findings, with some children found to be positively affected (i.e., high sense of self-concept and social competence) by having a sibling with ASD whilst others reported negative experiences (i.e., feelings of loneliness, lower levels of prosocial behavior, and increased internalising behaviors). These mixed findings are likely a result of diagnostic-related factors such as the severity of the ASD diagnosis, the degree of problematic and/or aggressive behavior expressed by the sibling with ASD, and the familial support given to the typically developing sibling. In addition, Orsmond, Kuo, and Seltzer (2009) also noted mixed findings indicating they could be the result of different sample characteristics (e.g., age ranges) across studies which may obscure age-related changes in the sibling relationship and sibling wellbeing. For example, typically-developing siblings have been found to show decreased satisfaction in their sibling relationship during adolescence and early adulthood; however, during middle to late adulthood siblings show increased satisfaction and contact. Furthermore, other subsystems within the broader family system can also influence the emotionality of typically developing siblings, with Meadan and colleagues (2010) concluding, (for example), that in conditions of greater marital stress, sibling relationships would suffer and harmful behaviors occur in sibling/ASD sibling interactions. Bitsika, Sharpley, and Mailli (2015), in a pilot study of the experiences of Australian siblings of an ASD-diagnosed family member (using interview responses), found that there were likely impacts across the family due to the demands of the situation. Furthermore, extensive study seemed likely to be useful and the current study therefore used a number of scales that enabled quantitative measures of the efficacy, affect and coping strategies used.

\subsection{Wider Impact on Typically Developing Siblings: Stigma}

For children, specifically for those in close age proximity to their sibling(s) with ASD, the experience of stigma may be overwhelming, resulting in depression, feelings of shame, and avoidance behaviors (Vidojevic, Gligorovic, \& Dragojevic, 2014). The typically developing sibling's connection to the child with ASD also 
impacts on how stigma emotionally affects them. Thus, victim stigmatisation does not only affect the child with ASD, but also everyone connected to that child: stigma causes further disruption to the family dynamics, leading to siblings avoiding social situations, actively hiding the condition from their peers, and resulting in some becoming victims of discrimination themselves [Vidojevic et al., 2014: see also Angell, Meadan, \& Stoner (2012) for a qualitative report on the responses of siblings touching on sibling cohesion, adaptability, coping strategies and personal techniques used in relation to the sibling with ASD]. Research has consistently shown that once a diagnosis of ASD occurs in a family, relationships with friends and extended family members deteriorate (Glasberg, 2002; Macks \& Reeve, 2007; Vidojevic et al., 2014). According to research by Macks and Reeve (2007), family members, specifically siblings, report depression, anxiety, and externalising behavioral problems in response to diminished social interactions, lack of social inclusion, lack of peer understanding, and lack of perceived emotional support.

\subsection{Psychological Wellbeing: Emotional and Social Efficacy and Affect}

Several studies have compared efficacy, affect, and coping strategies used in individuals with an ASD sibling versus individuals without an ASD sibling. The research that has been conducted in this area has revealed that having a child with ASD within the immediate family, places typically developing siblings at an increased risk of both externalising and internalising problems (Kaminsky \& Dewey, 2002; Vidojevic et al., 2014). In an earlier study conducted by Gold (1993), siblings of children with ASD were found to have higher self-reported levels of depression when compared to siblings of typically developing children. Furthermore, siblings of children with ASD reported high levels of loneliness and social problems when compared to siblings of typically developing children (Kaminsky \& Dewey, 2002). These findings are important, as children who experience social isolation and poor social relationships are at risk for social adjustment problems in adolescence and adulthood (Glasberg, 2000). Thus, these findings tend to support the notion that the psychological adjustment (emotional and social efficacy) of siblings of children with ASD is significantly poorer than for other children. These studies have not been replicated previously using an Australian sample directly studying siblings of children with ASD. We addressed this issue.

Poor psychological well-being also can be seen to stem from the social interactions that individuals have with their ASD sibling (Rivers \& Stoneman, 2003; Rodrigue, Geddken, \& Morgan, 1993). Sibling interactions are an important and powerful component of socialization, as they foster the development of instrumental social skills. Inconsistent sibling interactions, such as those experienced by a child exhibiting disabilities, may disrupt the development of psychological well-being (Meadan, Stoner, \& Angell, 2010; Rodrigue et al., 1993). Siblings of children with ASD are more susceptible to psychological maladjustment due to the experience of numerous daily stressors and inconsistent sibling relationships (Glasberg, 2000). These stressors include loss of parental attention, changes in the family dynamic, and stigma (Rodrigue et al., 1993). Furthermore, increases in parental stress may result in residual negative effects on other siblings within the family (Rodrigue et al., 1993). It also seems that parents especially mothers of children with ASD tend to engage in activities more frequently with their ASD child than with their typically developing child/children, in attempts to develop the social skills of their child with ASD (Rodrigue et al., 1993; Truchon, 2015). This may in turn have some impacts on the psychological adjustment (feelings of wellbeing as in positive affect, and the development of emotion regulation and social skills) of siblings of ASD-diagnosed individuals (Glasberg, 2000). Thus, the current study sought to compare levels of psychological well-being (as measured by efficacy, affect, and coping) between siblings of children with ASD and siblings of typically developing children, in an Australian based sample.

\subsection{Broader Autism Impacts (Phenotype: Including relation to Cognitive/Academic Efficacy)}

Further contributing to the psychological ill-health of siblings of children with ASD is the suggestion that a Broad Autism Phenotype may exist in first-degree relatives (Yirmiya et al., 2006). Evidence of a broader phenotype in ASD was first reported in the landmark twin study of Folstein and Rutter (1977). This study examined the cognitive and language abilities of monozygotic twin pairs where only one twin was diagnosed with ASD. Upon further examination of the "typically developing" twin, investigators detected increased rates of language and cognitive deficits, similar to those seen in ASD. These deficits included language delay, and inappropriate communication. Since this study by Folstein and Rutter (1977), several case-control familial studies have confirmed and extended these findings by documenting a range of subtle language, cognitive, social, and personality characteristics that parallel the diagnostic features of ASD (Bolton et al., 1994; Losh, Childress, Lam, \& Piven, 2008; Murphy et al., 2000). Interestingly, 25\% of siblings of children with ASD exhibit this phenotype of social and cognitive deficits in a subtle form (Oerlemans et al., 2013). It appears that siblings may not only experience the effects of their siblings' diagnoses, but also may experience difficulties themselves thus contributing to risk of poor social adjustment. Research has also identified differences in academic and cognitive 
functioning (see, for example, Gizzoni et al., 2014; Pajares \& Schunk, 2001; Yirmiya et al., 2006), psychological well-being, and emotionality (Gold, 1993; Kaminsky \& Dewey, 2001), when compared to siblings of typically developing individuals. There have, however, been inconsistencies in the reported studies regarding how siblings of ASD diagnosed family members respond. In light of the inconsistent research, the current study compared overall efficacy in social, emotional and academic terms, and also compared the overall adaptive and maladaptive coping methods reported in our Australian sample. Coping strategies are discussed next.

\subsection{Impact on Coping Strategies}

Ross and Cuskelly (2006) examined coping strategies used by siblings of children with ASD. They concluded that the most commonly used coping strategies in response to their siblings' aggressive behaviors were emotional regulation and wishful thinking. This suggests that siblings of children with ASD are aware of the levels of distress and anger that ASD aggressive behaviors produce in them and choose to cope with these situations by actively controlling their emotional reactions- due to recognition that the aggressive behaviors are illness related (Ross \& Cuskelly, 2006), and/or by escaping at least momentarily from the impacts of the situation. Whether these may be considered adaptive or maladaptive behaviors in their circumstances may need more extensive study. Certainly, research suggests the importance of family contexts in developing coping strategies that may be considered adaptive or maladaptive (cf., Davis, 2010; Hutton \& Caron, 2005; Trubia et al., 2016) and educational and support groups for siblings have been recommended and found effective (e.g., Smith \& Perry, 2006). However, no studies appear to have compared in general terms (adaptive, maladaptive) the coping strategies used by siblings of children with ASD and siblings of children without ASD. Thus, the current study included some attention to this aspect in the analyses (see Method for description of adaptive and maladaptive coping as assessed in the current study).

\subsection{Aims and Hypotheses of the Current Study}

We examined the wellbeing of ASD siblings (in terms of efficacy, affect, and coping strategies) in our Australian sample via the following hypotheses that were based on a review of the literature (above): it was hypothesised that:

I. Siblings of individuals with ASD would report lower levels of academic, social, and emotional efficacy than individuals with typically developing siblings.

II. Siblings of individuals with ASD would report lower levels of positive affect and higher levels of negative affect compared to siblings of individuals without ASD.

III. Siblings of individuals with ASD would report significantly more adaptive coping compared to siblings of typically developing children. However, given the limited studies to date examining such differences, and given that being in circumstances where coping was and is a vital concern, and where siblings are often stressed; it is recognised that there might be stronger maladaptive elements also among siblings of those with ASD. This hypothesis was kept more as an open question for preliminary investigation.

\section{Methods}

\subsection{Participants}

The original sample consisted of siblings in families with a child with diagnosed ASD, siblings in families with no ASD diagnoses, and parents of a child or children with ASD. The current article examined the sibling relationships. The final sample consisted of 156 Australian participants aged 6 to $52(M=21.06, S D=8.23)$, with 120 (77\%) being female. Eighty-two (82) participants (age range: 6 to 40) were siblings of an ASD-diagnosed individual while 74 participants (age range: 12 to 52) were siblings of typically developing individuals. Each sibling group were comprised of mostly females, with 63 females $(77 \%)$ in the group of siblings of an ASD-diagnosed person and 57 females (also 77\%) in the group of siblings of typically developing persons. More information is given under the Demographic Questionnaire sub-heading next.

\subsection{Materials}

\subsubsection{Demographic Questionnaire.}

The demographic questionnaire contained six basic questions regarding age, gender (male or female), number of siblings, whether the respondent was a sibling of a child with ASD, the level of functioning of the child with ASD, and the number of ASD siblings in the family (see Table 1). 
Table 1. Demographic Information

\begin{tabular}{llll}
\hline Dependent Variable & Siblings; ASD children & Siblings; non-ASD & Total \\
\hline Number & 82 & 74 & 156 \\
Age Range - years & $6-40$ & $12-52$ & $6-52$ \\
Mean (SD) Age - years & $16.89(5.89)$ & $22.51(4.93)$ & $21.06(8.23)$ \\
Number of Females: Males & $63: 19$ & $57: 17$ & $120: 36$ \\
Mean Number of Siblings & 1.89 & 1.95 & 1.92 \\
\hline
\end{tabular}

Of the 82 participants with ASD-diagnosed siblings, 72 individuals had one ASD-diagnosed sibling and 10 individuals had two siblings diagnosed. Additionally, 15 individuals reported that their sibling had mild severity ASD, 35 moderate severity ASD, and 32 severe ASD. Demographic statistics were identified for each group in terms of age and age ranges, and number of siblings. The Siblings (ASD) group age means (\& standard deviations) for age compared with the non-ASD sibling group are shown in Table 1, along with the similar numbers of siblings in each group. The age difference (16.89 to 22.51) was mainly attributable to the greater number of 6-11 year olds in the sample of individuals with an ASD-diagnosed sibling. There were 14 of the 82 individuals in this category ( $9 \%$ of the ASD sibling group). These children had their responses completed with their parent or carer to assist in accuracy of responses. We checked this difference in age range. Student t-tests showed there were no significant differences between the two groups for age $(p=0.062)$ or number of siblings $(p=0.36)$. We thus continued without modification to the sample membership in our analyses retaining all participants.

\subsubsection{The Self Efficacy Questionnaire for Children (SEQ-C; Muris, 2001).}

The SEQ-C measures three main areas of self-efficacy which are social, academic, and emotional. The questionnaire has been used across wide age ranges, e.g. with children and young adults. In our study older participants were asked to reflect on their childhood in answering the questions. Social self-efficacy refers to a child's ability to deal with social challenges. Academic self-efficacy refers to the perceived ability of the child to master academic affairs. Emotional self-efficacy refers to a child's perceived ability to resist peer pressure and engage in high-risk activities. The questionnaire is comprised of 24 questions with respondents rating their level of agreement with each question on a 5-point Likert-type scale $(1=$ Not at all to $5=$ Very well $)$. Total scores range from 24 to 120 and subscale scores (social efficacy, academic efficacy, and emotional efficacy) range 8 to 40. Consistent with existing literature, Cronbach's alpha coefficients for the total scale and the three subscales in the current study across all participants, ranged from $\alpha=.87$ to $\alpha=.89$, consistent with earlier results (Muris, 2001). The internal consistency responses indicated that the questionnaire worked well across the age ranges in our study (from 6 to 52 years of age).

\subsubsection{Positive and Negative Affect Schedule (PANAS; Watson, Clark, \& Tellegen, 1988).}

Positive and negative affect were measured by the PANAS scale, developed by Watson and colleagues (1988). The PANAS is a 20-item self-report questionnaire, with 10 items measuring positive affect (e.g., excited) and 10 items measuring negative affect (e.g., upset). Participants rated items on a 5-point Likert-type scale $(1=$ Very slightly or not at all to $5=$ Extremely) measuring the extent to which they have experienced positive and negative affect within a specified time period (i.e., the past week or the present moment). Total scores for positive affect and negative affect each range from 10 to 50, with higher scores indicating greater affect. The PANAS scale is a practical and brief way to measure two dominant dimensions within mood: Positive and negative affect (Watson et al., 1988). Consistent with existing literature (e.g., Crawford \& Henry, 2004; Watson et al., 1988), Cronbach's alpha for the PANAS in the current study were, for positive affect, $\alpha=.91$, and for negative affect, $\alpha=.87$. For participants under the age of 18 , we used the child version of the PANAS (PANAS-C) which also has sound validity for the younger age group.

\subsubsection{Brief COPE Inventory (Brief COPE; Carver, 1997).}

The Brief COPE is a 28 -item abbreviated version of the original 60 -item inventory and is often used when time constraints exist. Items of the scale can be categorised into subscales: such as adaptive and maladaptive coping, emotion-focused, problem-focused, and dysfunctional coping (Cooper, Katona, \& Livingston, 2008) though the original scale developers themselves do not support provision of subscales as being, say 'adaptive' or 'maladaptive', indicating that circumstances vary and what might be adaptive in some circumstances may not be adaptive in others - and that main attention should normally be given to the 14 sub-scales of coping that they identified. For the current study we have, however, used the adaptive-maladaptive approach that have been 
followed by several researchers in their examinations of coping responses in a variety of normal groups and groups where disabilities are evident (e.g., Brown, 2014; Davis, 2010; Wingsiong, 2015). The Brief COPE is made up of 14 subscales that describe coping strategies (e.g., using humor, removing oneself from a situation, reframing circumstances more positively, using prayer, being active) and these subscales can be grouped to form different categories of coping constructs (cf., Bose, Bjorling, Elfstrom, Persson, \& Saboonchi, 2015; Lai, Goh, Oei, \& Sung, 2014). Items were rated on a 4-point Likert-type scale $(1=$ I haven't been doing this at all to $4=$ I've been doing this a lot). We emphasised adaptive and maladaptive coping in this study with the following scales identified as adaptive coping (active coping, planning, positive re-appraisal, acceptance, emotional support, instrumental support, humor and religion) and the following subscales as maladaptive (venting, self-distraction, denial, substance use/abuse, behavioral disengagement and self-blame). Other studies have used different subscales, more commonly with humor and religion being in the 'maladaptive' list. In our analysis of the subscales humor and religion fell more readily in the adaptive or the neutral coping areas. Cronbach's alpha internal consistency coefficient for the full Brief COPE scale in the current study was $\alpha=.93$; for the adaptive coping subscale, $\alpha=.86$, and for the maladaptive coping subscale, $\alpha=.80$. Whether humor and religion were part of the adaptive or maladaptive categories (or not) made no significant difference to the internal consistencies of the scales. The adaptive and maladaptive coping composite scales correlated 0.41 with each other consistent with the overall scale emphasizing 'coping strategies' and explaining just over $16 \%$ of the variance in common - that is indicating overlap but with much also not in common (adaptive vs maladaptive).

\subsection{Procedure}

Before recruiting participants, the project gained ethical approval from the University's Human Research Ethics Committee. Once ethical approval was granted, a convenience sample was used whereby participants were recruited from the University's Psychology Student Participation portal and from social media outlets. No incentive was given for participation. The survey package received by each participant indicated that the study would take 30-60 minutes to complete. The order of presentation was consistent across all participants, beginning with the demographic questionnaire, followed by the PANAS, the SEQ, the Brief COPE Scale, and a final scale not reported in the current study, on strengths and difficulties faced. Each participant's responses were coded to maintain confidentiality and participants were assured that their questionnaire responses would remain confidential. It was explained that participation was voluntary and that the data would be stored for five years in the University. Data was collected by the researchers using both hard-copy and electronic questionnaires. Participants either arranged a time to meet the researcher at the University to collect a hard-copy, or logged onto an online server to complete the questionnaire. Participants who were emailed the questionnaire were given a week to return the completed questionnaire via email or post. Consent was requested for all participants. For participants under the age of 18, parental consent was obtained. For participants under the age of 13, parents / carers were requested to complete the questionnaire with their children to ensure that valid data was collected.

\subsection{Design}

In order to test the hypotheses, a one-way between-subjects Multivariate Analysis of Covariance (MANCOVA) was conducted to compare the three domains of self-efficacy (social, academic, and emotional), affect (positive and negative), and coping strategies (adaptive and maladaptive) while controlling for age. The age of participants was controlled for in the current study as this has been cited as possible reason for mixed findings in previous studies. In order to support the first hypothesis, siblings of children with ASD should report lower levels of self-efficacy across the three domains compared to siblings of children without ASD. Support for the second hypothesis would be evidenced by higher levels of negative affect and lower levels of positive affect in siblings of ASD children compared to siblings of typically developing children. Finally, to support the third hypothesis, siblings of children with ASD should show higher levels of adaptive coping compared to siblings of non-ASD children.

\section{Results}

\subsection{Data Cleaning and Initial Diagnostics}

Review of the initial dataset prior to analysis lead to a sample size of 156; however, 17 participants had not completed the efficacy scales and 37 had not completed the coping scales. Thus, the total sample size varied for each analysis where these scales were involved. However, assumptions for MANOVA were met. Furthermore, each sibling group was balanced with no significant difference found between cell sizes of siblings of an ASD child and siblings of a child without ASD; $\chi^{2}(1, N=156)=.41, p=.522$. Running a two-way MANOVA to compare sibling groups was considered; however, it was decided that it would be more beneficial to control for age as a continuous covariate in a one-way MANOVA. The current study, however, controlled for the potential cohort 
effects of age as there is insufficient literature to make specific hypotheses about the sibling relationship in early childhood (ages 6-12). Furthermore, the current study did not run a two-way analysis as student t-tests showed no significant difference between the mean ages of individuals in each sibling group.

The effects of the missing data were considered in the data cleaning stage. There was no trend by age across the efficacy and coping scales (that were not completed). Little's Missing Completely at Random (MCAR) Test was conducted with the completed efficacy and coping scales to determine whether there was systematic missing data that varied as a function of age. Little's MCAR test indicated participants' responses were missing completely at random; $\chi^{2}(1,3)=1.33, p=.723$. Thus, missing data, mainly on the efficacy and coping scales, were not considered likely to unduly affect the results of the study.

\subsection{Preliminary Analysis}

Pearson's bivariate correlations were conducted to test the assumption of multicollinearity. Table 2 shows means, standard deviations, and bivariate correlations between dependent variables. Bivariate correlations (all under $0.90)$ indicated that the assumption of multicollinearity $(<.90)$ was met and the one-way between-subjects MANCOVA could be conducted (Tabachnick \& Fidell, 2013). In line with previous research, all three components (i.e., academic, social, and emotional efficacy) were significantly positively correlated with one another. As expected, positive affect and negative affect were negatively correlated with one another; though adaptive and maladaptive coping were positively correlated with one another, consistent with in-common coping methods elements.

Table 2. Means, Standard Deviations, and Bivariate Correlations between Dependent Variables ( $\mathrm{N}=119$ - 156).

\begin{tabular}{|c|c|c|c|c|c|c|c|c|c|}
\hline Variable & $M$ & $S D$ & 1. & 2. & 3. & 4. & 5. & 6. & 7. \\
\hline 1. Academic Efficacy & 28.06 & 5.91 & - & & & & & & \\
\hline 2. Social Efficacy & 28.72 & 5.92 & $.55^{* * *}$ & - & & & & & \\
\hline 3. Emotional Efficacy & 24.17 & 6.01 & $.59 * * *$ & $.72 * * *$ & - & & & & \\
\hline 4. Positive Affect & 31.51 & 7.53 & $.34 * * *$ & $.55^{* * *}$ & $.47 * * *$ & - & & & \\
\hline 5. Negative Affect & 19.99 & 7.63 & $-.30 * *$ & $-.42 * * *$ & $-.56^{* * *}$ & $-.39 * * *$ & - & & \\
\hline 6. Adaptive Coping & 44.67 & 9.68 & .17 & $.20 *$ & .13 & $.21 *$ & .01 & - & \\
\hline 7. Maladaptive Coping & 13.06 & 3.48 & $-.19 *$ & $-.27 * *$ & $-.43 * * *$ & $-.19 *$ & $.37 * * *$ & $.35 * * *$ & - \\
\hline
\end{tabular}
$* p<.05 .{ }^{* *} p<.01 . * * * p<.001$.

To investigate the hypotheses, a one-way (Sibling Group: has an ASD sibling vs does not have an ASD sibling) between-subjects MANCOVA was conducted with efficacy, affect, and coping strategies while controlling for age. Box's M test of equality of covariances was met; $F(28,45461)=1.13, p=.289$. Levene's test of equality of error variances was violated for social efficacy and a more stringent alpha level of .01 was adopted for interpreting univariate analyses of social efficacy scores; $F(1,117)=5.15, p=.025$. As the assumptions were not severely violated, an alpha level of .05 was used to determine statistical significance and Wilk's lambda was reported for multivariate tests.

A significant multivariate effect of age on the dependent variables was found thereby justifying the inclusion of age as a covariate; $F(7,110)=2.52, p=.019$, partial $\eta^{2}=.138$, power $=.862$. At the univariate level, age had a significant effect on adaptive coping whereby adaptive coping varied as a function of age; $F(1,116)=12.15$, $p=.001$, partial $\eta^{2}=.095$, power $=.933$. However, there was a non-significant effect of age on affect scores, efficacy scores, and maladaptive coping scores.

Regarding the sibling group, there was a significant multivariate effect of sibling group on the dependent variables after controlling for age; $F(7,110)=3.03, p=.006$, partial $\eta^{2}=.162$, power $=.927$. Means and standard deviations for scores on the dependent variables in each sibling group are displayed in Table 3. Univariate analyses showed significant differences between sibling groups on social efficacy and emotional efficacy, with siblings of children with ASD displaying lower scores; $F(1,116)=6.19, p=.014$, partial $\eta^{2}=.051$, power $=.694$ and $F(1,116)=11.18, p=.001$, partial $\eta^{2}=.088$, power $=.912$ respectively. However, only partial support was provided for hypothesis one as no significant difference was noted between sibling groups on academic efficacy. Consistent with the second hypothesis, Table 3 shows that siblings of children with ASD displayed lower levels of positive affect and higher levels of negative affect compared to siblings of typically developing children; $F(1,116)=6.48, p=.012$, partial $\eta^{2}=.053$, power $=.714$ and $F(1,116)=4.17, p=.043$, partial $\eta^{2}=.035$, power $=.526$ respectively. Furthermore, univariate analyses showed no significant difference in 
the adaptive or the maladaptive coping strategies between sibling groups, thus not supporting hypothesis three that there would be more adaptive strategies in the ASD-sibling group.

Table 3. Means and Standard Deviations of Scores on Dependent Variables for Siblings of ASD Children and Siblings of Children without ASD

\begin{tabular}{|c|c|c|c|}
\hline \multirow[t]{2}{*}{ Dependent Variable } & \multicolumn{2}{|l|}{$\begin{array}{l}\text { Siblings of ASD children } \\
(n=82)\end{array}$} & $\begin{array}{l}\text { Siblings of non- } A S D \\
\text { children } \\
(n=74)\end{array}$ \\
\hline & $\mathrm{M}(S D)$ & & $\mathrm{M}(S D)$ \\
\hline Academic Efficacy & $27.42(6.38)$ & & $28.80(5.59)$ \\
\hline Social Efficacy & $27.12(6.19)$ & $*$ & $30.58(5.04)$ \\
\hline Emotional Efficacy & $22.23(5.45)$ & $* *$ & $26.42(5.88)$ \\
\hline Positive Affect & $29.73(7.77)$ & $*$ & $33.57(6.72)$ \\
\hline Negative Affect & $21.78(7.93)$ & * & $17.91(6.76)$ \\
\hline Adaptive Coping & $44.13(9.79)$ & & $45.31(9.68)$ \\
\hline Maladaptive Coping & $13.23(3.76)$ & & $12.85(3.14)$ \\
\hline
\end{tabular}

Note. $\mathrm{N}=156 . M=$ Mean. $S D=$ Standard Deviation. ${ }^{*} p<.05 .{ }^{* *} p<.01 .{ }^{* * *} p<.001$.

\section{Discussion}

The purpose of the current study was to investigate whether siblings of children with ASD differ in levels of efficacy, affect, and coping, compared to siblings of typically developing children. The study aimed to extend previous research by utilising an Australian-wide sample to extend the generalisability of past conclusions.

Hypothesis one, that ASD siblings would have lower levels of social, academic, and emotional efficacy when compared to siblings of typically developing individuals, was partially supported. The siblings of an ASD individual in the family reported significantly lower scores on emotional and social efficacy when compared to the typically-developing sibling group. These results are consistent with much research that indicates reduced skills and wellbeing demonstrated by siblings of those with ASD, as outlined earlier (e.g., Bitsika et al., 2015; Glasberg, 2000; Kaminsky \& Dewey, 2002; Macks \& Reeve, 2007; Meadan, Stoner, \& Angell, 2010; Orsmond et al., 2009). Our Australian sample fared similarly to the majority of previous studies indicating the pervasive negative effects that exist when family members suffer from severe disorders (cf., Davis, 2010; Truchon, 2015; Wingsiong, 2015)

However, still dealing with hypothesis one, no significant differences were found with regards to levels of academic efficacy. It was expected that academic efficacy would be lower in siblings of children with ASD as research has shown that a warm, responsive, and supportive home environment allows children to develop self-efficacy, related to academic efficacy. Previous research has suggested that due to the complex nature of ASD, siblings may receive reduced parental attention and support, which may lead to lower social, emotional, and academic self-efficacy in siblings (Macks \& Reeve, 2007; Nordone, 2014). Additionally, the Broad Autism Phenotype Theory had indicated that families of ASD children might be expected to display broad deficits in cognitive skills - which can lead to lower self-esteem among family members (Gizzonio, Avanzini, Fabbri-Destro, Campi, \& Rizzolatti, 2014; Yirmiya et al., 2006). However, the current findings may possibly be explained by other research suggesting that similar age peers can influence the development of academic self-efficacy via model similarity (Pajares \& Schunk, 2001). For example, watching similar aged peers at school succeed may increase a child's academic self-efficacy and motivate them to complete their school work. Thus, regardless of whether the child is receiving parental attention at home, watching similar aged peers succeed at school may in turn increase their own perception of their abilities to succeed in academic domains.

The second hypothesis, that lower levels of positive affect and higher levels of negative affect would be evident in the ASD sibling group, was fully supported. No current literature was identified that analysed ASD siblings in relation to affect using the PANAS, but the findings of the current study were theoretically consistent with Family Systems Theory and in line with expectations from related research (e.g., on depression and wellbeing: see Davis, 2010; Gold, 1993; Kaminsky \& Dewey, 2002; Macks \& Reeve, 2007; Orsmond et al., 2009). As minimal research exists regarding affect and ASD siblings, this finding contributes new information on the understanding of the impacts of an ASD diagnosis on siblings. This finding is also consistent with existing 
literature highlighting that siblings of children with ASD are more susceptible to psychological maladjustment due to the experience of numerous daily stressors and inconsistent sibling relationships (Glasberg, 2000; Rodrigue et al., 1993). The use of the PANAS to assess the mood states in our sibling study has added a further dimension to the studies available on affect.

The third hypothesis, that siblings of children with ASD would report significantly more adaptive coping compared to siblings of typically developing children, was not supported. There were no significant differences found for either the adaptive or the maladaptive coping strategy groups. The hypothesis was based on the observation that children brought up in difficult circumstances may learn adaptive strategies (one of the aims of coping programs and support groups- cf., Smith \& Terry, 2005), or perhaps equally, might develop psychological difficulties themselves and thus use maladaptive strategies more often than individuals in typically developing families (numerous studies indicating negative effects from having to deal with difficult family situations, as described above and also seen in our study). There was no difference. It could be that the selected combination of subscales into adaptive and maladaptive coping strategies may have masked differences in some of the 14 strategies used but this was not examined for the current paper. If siblings in families with a diagnosed ASD individual are not employing additional or more coping strategies beyond those of normal sibling families, this may be seen as a positive, given that it might have been expected that there would be negative outcomes from the circumstances faced. That is, siblings who had already in our sample been identified as suffering from reduced capacities in emotional and social efficacy displayed equally effective (or ineffective) strategies as did those from typically developing families. This area of coping has in general been well researched, but not with the use of the brief COPE and its listing of specific strategies. The general studies on coping have included those by Hesse et al. (2013), Kaminsky and Dewey (2002), and Ross and Cuskelly (2006). Given that just two groupings were considered (adaptive and maladaptive coping strategies) there is room for more research on the coping strategies used in the ASD related studies. For example, several of the studies using the Brief Cope have applied different models (or groupings of the 14 strategies) to other disability areas (e.g., Bose et al., 2015; Cooper et al., 1997).

\subsection{Limitations and Sampling Issues}

Although the results of the present study have implications for monitoring the psychological well-being of ASD siblings, there were several aspects of the sample that should be considered when interpreting the study's findings. Firstly, there was a substantial attrition rate, with many participants not completing the last questionnaire. Future research should consider administering the questionnaires in a fully randomised order to ensure a counter-balanced design and reduce systematic attrition. Additionally, the current study examined efficacy, affect, and coping as indicators of overall psychological wellbeing in siblings. However, future research may want to utilise specific psychological well-being scales to specifically measure the construct of psychological well-being. Furthermore, the current study did not use a social desirability scale and was unable to detect socially desirable responding from participants. Future research may benefit from using a social desirability scale to detect potentially biased responding. Finally, the current study used similar questionnaires across a wide age range (the questionnaires were modified either in wording or in appropriate presentation- e.g. with parental assistance): the impacts of these differences are not known though the aim of the strategies adopted was to enable the trends in answers to become apparent. Further studies within age groups alone would be helpful. We believe the trends and implications are clear from our study- as indicated next.

Findings in the current study suggest that siblings of children with ASD show greater levels of negative affect and lower levels of positive affect compared to siblings of typically developing children. Additionally, our findings suggest for our sample that siblings of children with ASD show lower levels of social and emotional self-efficacy (indicators of self-esteem) compared to the typically developing siblings. Taken together, these findings show that family characteristics (e.g., having a child with ASD in the family) may result in altered sibling relationships, thereby affecting the development of psychological wellbeing (comprising of affect and efficacy) in the neurotypical, normally developing, siblings (Glasberg, 2000; Rodrigue et al., 1993). Due to the complex nature of ASD and its associated social and communication difficulties, typically developing siblings would be affected more, hence showing the significantly higher levels of negative affect and lower levels of positive affect. Family Systems Theory posits that boundaries exist between family subsystems (e.g., sibling relationships) and the outside world and these systems can influence one another (Cridland et al., 2013). Previous research has suggested that the combination of reduced parental support and attention as well as perceived stigma towards the child with ASD, may become overwhelming for the typically developing sibling (Vidojevic et al., 2014). This combination of internal and external stressors can impact the psychological well-being of siblings of children with ASD. Thus, it is important for future studies to replicate this research and 
examine how interventions can elevate levels of positive affect, social efficacy, and emotional efficacy in siblings of children with ASD. The use of sibling support groups (e.g., Smith \& Perry, 2006) recommended more than a decade ago and other strategic interventions that encourage personal development in the areas most strongly affected can be strongly supported.

\subsection{Implications}

The findings of the current study indicate that ASD siblings have lower overall levels of psychological well-being than siblings of typically developing individuals. Specifically, this reduced well-being was demonstrated via reports showing reduced social efficacy and emotional efficacy as well as decreased positive affect and increased negative affect in siblings of ASD-diagnosed family members vs siblings in typically developing families. Despite the limitations, the current study has indicated that siblings of those diagnosed with ASD are affected by the circumstances in which they have found themselves. While some theories might suggest that we 'broaden and build' from severe difficulties and/or that we can find meaning and enrichment in life through the difficulties experienced, this was not the experience of most of the sibling respondents in the current study. Instead, help in achieving more positive outcomes would appear to be needed. These observations are consistent with those from many earlier studies suggesting the importance of support and interventions for families and their members (e.g., Bitsika et al., 2015; Giallo et al., 2014; Smith \& Perry, 2006).

Future research could explore these factors and investigate the effectiveness of sibling support groups as well as family support groups. The current study has provided further evidence on the impacts on family members when siblings have ASD. It is hoped that these findings will help underpin further development of needed support strategies and interventions for siblings of children with ASD, promoting their emotion regulation, and their social, academic and coping skills.

\section{Acknowledgments}

There were no special funding support arrangements made in regards to the project reported in this paper.

\section{References}

American Psychiatric Association. (2013). Diagnostic and statistical manual of mental disorders (5th ed.). Washington, DC: Author. https://doi.org/10.1176/appi.books.9780890425596

Angell, M. E., Meadan, H., \& Stoner, J. B. (2012). Experiences of siblings of individuals with autism spectrum disorders. Autism Research and Treatment, Article ID- 949586. https://doi.org/10.1155/2012/949586

Bitsika, V., Sharpley, C., \& Mailli, R. (2015). Experiences of Australian siblings of an individual with an autism spectrum disorder. Child and Family Behavior Therapy, 37(2), 93-14. https://doi.org/10.1080/07317107.2015.1035972

Bolton, P., Macdonald, H., Pickles, A., Rios, P., Goode, S., Bailey, A., \& Rutter, M. (1994). A case-control family history study of autism. Journal of Child Psychology and Psychiatry, 35, 877-900. https://doi.org/10.1111/j.1469-7610.1994.tb02300.x

Bose, C.N., Bjorling, G., Elfstrom, M., Persson, H., \& Saboonchi, F. (2015). Assessment of coping strategies and their associations with health related quality of life in patients with chronic heart failure: the brief COPE restructured. Cardiology Research, 6(2), 239-248. https://doi.org/10.14740/cr385w

Brown, S. (2014). Coping, resolution and advocacy in parents of children with autism. Degree thesis, Purchase College, State University of new York. New York.

Carver, C. S. (1997). You want to measure coping but your protocol's too long: Consider the Brief COPE. International Journal of Behavioral Medicine, 4, 92-100. https://doi.org/10.1207/s15327558ijbm0401_6

Cooper, C., Katona, C., \& Livingston, G. (2008). Validity and reliability of the brief COPE in carers of people with dementia: the LASER-AD Study. Journal of Nervous and Mental Disease, 11, 838-843. https://doi.org/10.1097/NMD.0b013e31818b504c

Crawford J.R., \& Henry, J.D. (2004). The Positive and Negative Affect Schedule (PANAS): Construct validity, measurement properties and normative data in a large non-clinical sample. British Journal of Clinical Psychology, 43, 245-265. https://doi.org/10.1348/0144665031752934

Cridland, E.K., Jones, S.C., Magee, C.A., \& Caputi, P. (2014). Family-focused autism spectrum disorder research: A review of the utility of family systems approaches. Autism, 18, 213-222. https://doi.org/10.1177/1362361312472261 
Davis, K.E. (2010). The psychological adjustment of siblings of children with disabilities: The role of the family and the wider social community. Doctor of Psychology thesis: Royal Melbourne Institute of Technology (RMIT): Melbourne.

Folstein, S., \& Rutter, M. (1977). Infantile autism: A genetic study of 21 twin pairs. Journal of Child Psychology and Psychiatry, 18, 297-321. https://doi.org/10.1111/j.1469-7610.1977.tb00443.x

Giallo, R., Roberts, R., Emerson, E., Wood, C., \& Gavidia-Payne, S. (2014). The emotional and behavioral functioning of siblings of children with special health care needs across childhood. Research in Developmental Disabilities, 35(4), 814-825. https://doi.org/10.1016/j.ridd.2014.01.017

Gizzonio, V., Avanzini, P., Fabbri-Destro, M., Campi, C., \& Rizzolatti, G. (2014). Cognitive abilities in siblings of children with autism spectrum disorders. Experimental Brain Research, 232, 2381-2390. https://doi.org/10.1007/s00221-014-3935-8

Glasberg, B.A. (2000). The development of siblings' understanding of autism spectrum disorders. Journal of Autism and Developmental Disorders, 30, 143-156. https://doi.org/10.1023/A:1005411722958

Gold, N. (1993). Depression and social adjustment in siblings of boys with autism. Advocate: The Newsletter of the Autism Society of America, 23, 147-163. https://doi.org/10.1007/BF01066424

Griffith, G.M., Hastings, R.P., \& Petlas, M.A. (2014). Brief report: Fathers' and mothers' ratings of behavioral and emotional problems in siblings of children with autism spectrum disorder. Journal of Autism and Developmental Disorders, 44, 1230-1235. https://doi.org/10.1007/s10803-013-1969-6

Hesse, T.L., Danko, C.M., \& Budd, K.S. (2013). Siblings of children with autism: Predictors of adjustment. Research in Autism Spectrum Disorders, 7, 1323-1331. https://doi.org/10.1016/j.rasd.2013.3.07.024

Hutton, A.M., \& Caron, S.L. (2005). Experiences of families with children with autism in rural New England. Focus on Autism and Other Developmental Disabilities, 20, 180-189. https://doi.org/10.1177/10883576050200030601

Kaminsky, L., \& Dewey, D. (2002). Psychosocial adjustment in siblings of children with autism. Journal of Child Psychology and Psychiatry, 43, 225-232. https://doi.org/10.1111/1469-7610.00015

Kovshoff, H., Cebula, K., Tsai, H-W. J., \& Hastings, R. P. (2017). Siblings of children with autism: The Siblings Embedded Systems Framework. Current Development Disorders Reports, 4(2), 37-45. https://doi.org/10.1007/s40474-017-0110-5

Lai, W.W., Goh, T.J., Oei, T.P.S., \& Sung, M. (2015). Coping and well-being in parents of children with autism spectrum disorders (ASD). Journal of Autism and Developmental Disorders, 45, 2582-2593. https://doi.org/10.1007/s10803-015-2430-9

Linley, P.A., Maltby, J., Wood, A.M., Osborne, G., \& Hurling, R. (2009). Measuring happiness: The higher order factor structure of subjective and psychological well-being measures. Personality and Individual Differences, 47, 878-884. https://doi.org/10.1016/j.paid.2009.07.010

Losh, M., Childress, D., Lam, K., \& Piven, J. (2008). Defining key features of the broad autism phenotype: a comparison across parents of multiple and single incidence autism families. American Journal of Medical Genetics Part B, 147B, 424-433. https://doi.org/10.1002/ajmg.b.30612

Macks, R.J., \& Reeve, R.E. (2007). The adjustment of non-disabled siblings of children with Autism. Journal of Autism and Developmental Disorders, 37, 1060-1067. https://doi.org/10.1007/s10803-006-0249-0

Meadan, H., Stoner, J.B., \& Angell, M.E. (2010). Review of literature related to the social, emotional, and behavioral adjustment of siblings of individuals with autism spectrum disorder. Journal of Developmental and Physical Disabilities, 22, 83-100. https://doi.org/10.1155/2012/949586

Muris, P. (2001). A brief questionnaire for measuring self-efficacy in youths. Journal of Psychopathology and Behavioral Assessment, 23, 145-149. https://doi.org/10.1023/A:1010961119608

Murphy, M., Bolton, P.F, Pickles, A., Fombonne, E., Piven, J., Rutter, M. (2000). Personality traits of the relatives of autistic probands. Psychological Medicine, 30, 1411-1424. https://doi.org/10.1017/S0033291799002949

Nordone, L. M. (2014). Sibling relationships: growing up with a sibling diagnosed with autism spectrum disorder and its influence on perceived family cohesion, attachment and current life satisfaction (master's thesis). Retrieved from https://scholarworks.smith.edu/cgi/viewcontent.cgi?article=1887\&context=theses. 
Oerlemans, A. M., Droste, K., van Steijn, D. J., de Sonneville, L. M., Buitelaar, J. K., \& Rommelse, N. N. (2013). Co-segregation of social cognition, executive function and local processing style in children with ASD, their siblings and normal controls. Journal of Autism and Developmental Disorders, 43(12), 2764-78. https://doi.org/10.1007/s10803-013-1807-x

Orsmond, G. I., Kuo, H. Y., \& Seltzer, M. M. (2009). Siblings of individuals with an autism spectrum disorder: Sibling relationships and wellbeing in adolescence and adulthood. Autism, 13, 59-80. https://doi.org/10.1177/1362361308097119

Pajares, F., \& Schunk, D. (2001). The development of academic self-efficacy. Development of achievement motivation, 7, 1-27. Retrieved from https://www.uky.edu/ eushe2/Pajares/SchunkPajares2001.PDF.

Rivers, J.W., \& Stoneman, Z. (2003). Sibling relationships when a child has autism: Marital stress and support coping. Journal of Autism and Developmental Disorders, 33, 383-394. https://doi.org/10.1023/A:1025006727395

Rodrigue, J.R., Geffken, G.R., \& Morgan, S.B. (1993). Perceived competence and behavioral adjustment of siblings of children with autism. Journal of Autism and Developmental Disorders, 23, 665-674. https://doi.org/10.1007/BF01046108

Ross, P., \& Cuskelly, M. (2006). Adjustment, sibling problems and coping strategies of brothers and sisters of children with autism spectrum disorder. Journal of Intellectual and Developmental Disability, 31, 77-86. https://doi.org/10.1080/13668250600710864

Smith, T., \& Perry, A. (2005). Support group for brothers and sisters of children with autism. Journal of Developmental Disabilities, 11, 77-88. Retrieved from http://citeseerx.ist.psu.edu/viewdoc/download?doi=10.1.1.473.566\&rep=rep1\&type=pdf

Tabachnick, B.G., \& Fidell, L. (2013). Using Multivariate Statistics $\left(6^{\text {th }}\right.$ ed.). New York, NY: Pearson Education.

Trubia, G., Buono, S., Panerai, S., Zingale, M., Passanisis, A., Pirrone, C., \& Di Nuovo, S. (2016). Siblings' perceptions in autism spectrum disorder compared with intellectual disability and typical development. Clinical Neuropsychiatry, 13(2), 10-16.

Truchon, M.M. (2015). Parental differential treatment and sibling relationship quality in families with a child with a developmental disability. (Unpublished Masters Dissertation), Faculty of Arts and Science, Concordia University, Montréal, Canada.

Vidojevic, I., Gligorovic, M., \& Dragojevic, N. (2014). Tendency towards stigmatization of families of a person with autistic spectrum disorders. International Journal of Social Psychiatry, 60(1), 63-70. https://doi.org/10.1177/0020764012463298

Watson, D., Clark, L.A., \& Tellegen, A. (1988). Development and validation of the brief measures of positive and negative affect: The PANAS Scales. Journal of Personality and Social Psychology, 54, 1063-1070. https://doi.org/10.1037//0022-3514.54.6.1063

Wingsiong, A.C. (2015). Parentification, coping, and distress in siblings of individuals with and without attention deficit / hyperactivity disorder (ADHD). $\mathrm{PhD}$ thesis: Electronic theses and Dissertations, 5468. University of Windsor. Downloaded from: https://scholar.uwindsor.ca/etd/5468.

Yirmiya, N., Gamliel, I., Pilowsky, T., Feldman, R., Baron-Cohen, S., \& Sigman, M. (2006). The development of siblings of children with autism at 4 and 14 months: Social engagement, communication, and cognition. Journal of Child Psychology and Psychiatry, 47, 511-523. https://doi.org/10.1111/j.1469-7610.2005.0152

\section{Copyrights}

Copyright for this article is retained by the author(s), with first publication rights granted to the journal.

This is an open-access article distributed under the terms and conditions of the Creative Commons Attribution license (http://creativecommons.org/licenses/by/4.0/). 